\title{
ENLÈVEMENTS DE STATUES DIVINES EN BORD DE MER : DE L'ARTÉMIS TAURIQUE À HÉRA REINE
}

\author{
Abduction of divine statues on the sea-shore: \\ from Taurian Artemis to Hera the Queen ${ }^{1}$
}

Pierre Ellinger ${ }^{2}$

\begin{abstract}
RÉSUMÉ
Cette étude examine, en les comparant, plusieurs traditions légendaires ou mythiques grecques, connues et moins connues, d'enlèvements de statues divines dans un sanctuaire en bord de mer : principalement, l'histoire de l'Artémis Taurique, dans sa version euripidéenne, sur fond de ses autres versions, à travers l'Antiquité, dans l'espace méditerranéen ; celle de l'enlèvement manqué de l'Héra de Samos, et la tradition de la fondation de la cité de Lyktos en Crète. L'attention est portée sur la dimension maritime du réseau de ces récits et sur le jeu de leur articulation commune autour de points nodaux que sont l'embarquement et l'appareillage, le débarquement et le mouillage, tout en tenant compte de leurs contextes d'énonciation. Il est possible ainsi d'avancer dans leur compréhension pour montrer comment, dans différents contextes historiques, ils ont $\mathrm{pu}$, entre autres, servir à justifier l'appropriation de l'espace maritime par divers pouvoirs antiques, entre commerce, piraterie et ambitions thalassocratiques.
\end{abstract}

Mots-clefs : Histoire légendaire; piraterie; thalassocratie; statues divines; imaginaire maritime.

\begin{abstract}
This paper aims at comparing several Greek legendary or mythical stories concerning the abductions of divine statues from seaside sanctuaries: the legends about Taurian Artemis, Euripides' version in the first place, with
\end{abstract}

1 Tous mes remerciements à Lorena Lopes da Costa et à Erica Angliker qui m'ont donné l'occasion d'écrire cette contribution, et pour leurs remarques et leur aide pour sa mise au point définitive, en particulier dans cette période difficile de pandémie.

2 Professor Emeritus of Ancient Greek History at Université de Paris. E-mail: pierre.ellinger@sfr.fr 
its background of other versions throughout the Mediterranean space in Antiquity; Samian Hera's failed abduction; and the tradition of the foundation of the Cretan city of Lyktos. We consider this network of tales in its maritime dimension and articulation around nodal points of sea-travelling, such as departure and landing, and in their enunciation context. Thus we get a better understanding of how, at different times, those tales justified ancient powers in their appropriation of maritime space, between commerce, piracy and thalassocratic ambitions.

Keywords: Legendary history; piracy; thalassocracy; divine statues; maritime imaginary.

Dans les récits légendaires grecs anciens, les sanctuaires de frontière des cités sont des lieux exposés, ceux des frontières terrestres comme des frontières maritimes. Dans les montagnes de l'intérieur, comme dans les prairies de bord de mer, voire quand elles courent pour Dionysos sur les montagnes côtières, femmes et jeunes filles sont menacées de viol ou d'enlèvement. Trop souvent, la lecture de ce type d'histoires s'est réduite au paradigme initiatique, et pour celles qui se déroulent sur les confins maritimes, le rôle qu'y joue la mer a été négligé ${ }^{3}$. Pourtant, par exemple, lorsque Solon provoque une agression mégarienne contre les femmes d'Athènes au sanctuaire de Déméter du cap Kolias, en substituant à celles-ci des jeunes gens déguisés en femmes, le but est bien de se saisir du navire des ennemis, et en se faisant passer pour eux et leurs captives, de franchir le détroit sans être reconnus, pour s'emparer de l'île de Salamine, en face (Plutarque, Solon, 8-9). Quand les premiers colons thraces de Naxos, uniquement des hommes, viennent, pour se procurer des femmes, ravir les bacchantes dans la montagne de la côte thessalienne, c'est bien le caractère insulaire de leur conquête, dans une Égée primitive pratiquement déserte, elle et ses îles, qui commande le récit (Diodore de Sicile, V, 50).

Un autre groupe apparenté d'histoires met en scène l'enlèvement de la statue divine, avec un rôle généralement important donné à la prêtresse qui en avait la charge. Principalement les récits autour de l'Artémis Taurique, pas seulement la tragédie d'Euripide. On y ajoutera les errances d'un autre

3 C'est le cas, par exemple, de LEITAO, 1999 (malgré le titre : « Solon on the beach »). Pour un retour critique et historiographique sur le modèle initiatique, cf. CALAME, 2010. 
de ses avatars à travers l'Égée, de l'Attique à Lemnos, puis au cap Ténare, à Sparte et finalement en Crète, où la déesse fonde l'une des plus redoutables cités guerrières de l'île. En contraste, un enlèvement manqué, celui de la statue d'Héra à Samos. La dimension maritime de ces histoires, qui a souvent échappé pour chacune prise isolément, ressort si on les considère comme un ensemble. Il faut pour cela renverser notre perspective accoutumée sur l'espace grec, en substituant à notre vision depuis le centre de la cité vers ses marges, un regard dirigé de la mer vers la terre. Les moments où on la quitte et où on la rejoint, éventuellement pour la conquérir, apparaissent alors comme des articulations essentielles de récits renvoyant à des situations historiques qui fluctuent au rythme de l'ordre et du désordre en Méditerranée.

\section{De la Tauride à l'Attique}

L'histoire de l'Artémis Taurique, enlevée par Oreste, tire sa force d'être à double titre paradoxale. D'abord, cet enlèvement, à la fois de la déesse et de sa prêtresse, est pieux, ordonné par Apollon, pour qu'Oreste soit guéri de sa folie. Deuxièmement, il ne se situe pas aux marges d'une cité grecque, mais à celles d'un monde barbare, au-delà d'une mer hostile, et même la Mer Hostile (pontos axeinos) par excellence (Euripide, Iphigénie en Tauride 4 , 124-125, 218-219, 438, etc.). Sur le rivage de ce pays extrême se pratique pour Artémis un rituel extrême, le sacrifice humain des marins et commerçants grecs qui ont réussi à y aborder ou y ont fait naufrage (I.T., 28-41, 69-75, 401-406 ; cf. Hérodote, IV, 103)5. Dans l'Iphigénie en Tauride $\mathrm{d}^{\prime}$ Euripide, l'espace maritime joue un rôle fondamental ${ }^{6}$. Plusieurs fois, la pièce évoque le trajet aller et retour d'Oreste : le périlleux franchissement des roches Symplégades, à la sortie du Bosphore, censées encore broyer entre elles les navires (I.T., 124-125, 241-242, 422) ; le choix décisif de la route du Pont Gauche, entre longer la redoutable côte rocheuse de Salmydessos,

4 Dorénavant $I . T$.

5 Sur la question de la réalité de ces sacrifices et du rapport autour d'eux des Grecs et des Taures, cf. USTINOVA, 1999, p. 98-99, et surtout BRAUND, 2018, p. 34-38, 51-53.

6 Voir le commentaire de KYRIAKOU, 2010, p. 10, et l'étude novatrice de Barbara KOWALZIG, 2013. 
sous les vents du Nord, ou la traversée directe jusqu'à Leuké, l'île Blanche d'Achille, d'où l'on peut piquer à l'Est vers la Tauride. Le chœur se réfère à l'expérience des marchands-navigateurs de l'époque classique (I.T., 408438 ; KOWALZIG, 2013, p. 183-190). Les héros arrivés au but du voyage, la topographie de la côte passe au premier plan. Le temple de la déesse dominant la falaise (I.T., 1196), la grotte marine, refuge des pêcheurs de pourpre, où Oreste et Pylade se cachent en contrebas de la côte, dont descendent, pour baigner leur troupeau de bœufs, les bergers qui vont les découvrir et les faire prisonniers (I.T., 106-109, 260-335). Enfin la crique à l'écart où est embusqué le navire, vers laquelle se dirige la procession guidée par Iphigénie, sous le prétexte de purifier les deux hommes, avant de les sacrifier, et la statue qu'ils auraient contaminée de leur épouvantable souillure (I.T., 1191-1201, 13271328, 1373-1376 : escarpements autour du rivage). C'est là que se passe la scène la plus dramatique, l'embarquement, particulièrement appréciée plus tard des sculpteurs de sarcophages et monuments funéraires de l'époque impériale $^{7}$ : le combat sur le rivage, Oreste qui porte dans la mer, sur son épaule, sa sœur et la statue (I.T., 1379-1385). Puis le navire tente en vain, à force de rames, de franchir pour sortir du port l'énorme vague que pousse subitement un violent vent contraire et qui menace de le fracasser en les rejetant sur les rochers (I.T., 1390-1419). L'apparition d'Athéna arrête les velléités de poursuite de Thoas, le roi des Taures. Un moment, ses hommes ont cru reconnaitre l'action de Poséidon dans le vent et les vagues s'opposant à la fuite du bateau (I.T., 1414-1418). Lui-même - peut-on supposer pensait l'invoquer quand il envoyait cavaliers et navires ${ }^{8}$ capturer les Grecs, au cas où ils seraient jetés à la côte, ou parviendraient à prendre le large (I.T., 1423-1430). Mais Poséidon, au contraire, se révèle l'allié de la déesse et le dieu poliade d'Athènes qu'il est avec elle. La navigation de retour se fera donc sans histoire grâce à leur bonne entente. Poséidon aplanit la mer, les Symplégades sont oubliées, Athéna escorte elle-même, sur le navire, la statue de sa sœur Artémis jusqu'à la côte orientale de l'Attique (I.T., 1444-

7 Cf. LIMC, V, 1 (1990), s.v. Iphigeneia, p. 723-726, n 68-82 (P. Linant de Bellefonds) ; BIELFELDT, 2005, p. 186-187, 217-221. La statuette, portée par Iphigénie, est alors en général représentée comme complètement enveloppée de linges, ne laissant apparaître éventuellement que le visage (sarcophage de Marseille, Iphigeneia $\left.\mathrm{n}^{\circ} 70\right)$.

8 En tant donc, à la fois, que Poséidon Hippios, des chevaux, et dieu de la mer. Thoas invoque aussi Artémis pour cette chasse, thèrasete, aux fugitifs, I.T., 1425-1426. Iphigénie inversement l'a priée pour qu'elle les sauve, sôson, 1402). 
1445 : Poséidon ; 1488-1491 : Athéna). Oreste installera celle-ci, avec son nouveau rituel pour les jeunes Athéniens, à Halai - où elle prendra le titre de Tauropolos, en souvenir de la Tauride -, directement au-dessus de la plage. Iphigénie ira poursuivre son office de prêtresse, pour les femmes, au sanctuaire jumeau de la déesse, quelques kilomètres plus au sud, au fond de la crique de Brauron. Deux sanctuaires côtiers à nouveau (I.T., 1449-1467. Cf. GUARISCO, 2015).

\section{Artémis Taurique des tempêtes}

Ce retour apaisé sur la mer débouche sur un rituel apaisé pour la déesse, désormais intégrée aux cultes civiques d'Athènes. On n'égorgera plus de victimes humaines pour elle, mais on se contentera de faire couler quelques gouttes de sang du cou d'un jeune homme en l'effleurant de la pointe d'une épée (I.T., 1458-1461). Or il n'en est pas de même dans les autres versions de l'histoire, là où nous avons des renseignements sur les modalités du retour. Loin de bénéficier d'une navigation tranquille, Oreste, Iphigénie et la statue sont jetés sur les diverses côtes de la Méditerranée par la tempête. La mer n'est pas apaisée par Poséidon, mais la déesse les sauve du naufrage dans une mer démontée. Ainsi quand ils débarquent à Rhodes (Apollodore, Epitomé, 6, 27) ${ }^{9}$, en Sicile (Servius, Comm. in Verg. Buc. Vol. III, p. 1, 9-12 Thilo = Scholia in Theocritum Vetera, Wendel, p. 20, 29-33) ${ }^{10}$ ou en Syrie (Tzetzès, Scholies à Lycophron, 1374) ${ }^{11}$. On recoupe ici un thème du héros projeté par la tempête sur des rivages lointains ou inconnus, fréquent dans les Nostoi (Ulysse, Ménélas, etc.) et les récits de découvertes et de fondations coloniales (Thouclès à Naxos de Sicile [Strabon, VI, 2, 2], Kolaios à Tartessos [Hérodote, IV, 152], les Phocéens en Gaule [Denys

9 « Mais certains disent qu'Oreste fut poussé par une tempête (vers Rhodes) et que $<$ la statue $>$ fut consacrée, conformément à un oracle, dans le mur d'enceinte. »

10 ad Siciliam ... tempestate delatus.

11 «Poussés par la tempête ils s'échouèrent sur le rivage (cheimasthentes de exôkeilan) des contrées où se trouve la ville appelée aujourd'hui Séleucie, au pays d'Antioche et du mont Mélantion ; ce mont, après qu'Oreste y eut été délivré de sa folie (mania), prit le nom d'Amanus (« sans folie »)»; SALIOU, 2006, p. 73-77. 
d'Halicarnasse, Antiquités romaines, XIV, 1, 3 $]^{12}$ ). Nous avons affaire alors à l'Artémis de la mer, celle de l'embarquement et du débarquement (Ekbatèria, Apobatèria), qui guide (Hègemonè) et sauve (Sôteira) dans les traversées lointaines et périlleuses (ELLINGER, 2009, p. 22-26 ; KOWALZIG, 2013, p. 186 ; FENET, 2016, p. 189-218), Nélée en route pour l'Ionie (ELLINGER, 2012, p. 227-229), les Phocéens vers Marseille (MALKIN, 2011, p. 182184). L'Artémis qu'Agamemnon invoquait à Mégare avant son départ pour Troie et que priera le poète Théognis pour qu'elle le préserve à son tour d'une navigation aux dangers mortels (Théognis, 11-14). Au IV siècle de notre ère, le rhéteur Libanius semble garder encore indirectement souvenir de cette action quand il utilise la métaphore maritime pour décrire la protection que la déesse apporte aux femmes pendant la grossesse et au moment de l'accouchement, qu'il compare à une tempête :

L'ardeur d'Aphrodite, qui n'existe qu'en vue des enfants, parvient à son but grâce à Artémis, de même que, lorsqu'on navigue sur les mers, on arrive à destination grâce aux ports. S'il n'y avait de ports nulle part, s'il n'y avait rien pour accueillir les navigateurs, la traversée serait stérile, et les navires se briseraient sur les écueils. Ainsi cette déesse est un port au bout de la traversée dans le ventre des mères (Libanius, Discours $\mathrm{V}$ [Artémis], 28 ; cf. 26 , pour ceux qui sont morts dans cette tempête, ou nés infirmes, faute de la présence de la déesse) $)^{13}$.

On a remarqué que les cultes de l'Artémis Tauropolos et de la Parthénos, la déesse qui reprend, à l'époque historique, dans la colonie grecque de Chersonésos de Crimée, l'héritage de l'Artémis Taurique du mythe (BRAUND, 2007 ; 2018, p. 15-95), se situent le long des voies du

12 « Leurs navires, emportés par des vents violents, abordèrent (ekelsan) dans le golfe Galate ». D'où le nom de Kelsikè, qui serait devenu ensuite celui de «Celtique », donné par eux au pays, quand, sauvés, ils atteignirent le rivage : « là où nous avons abordé poussés par la tempête ». Comparer avec la formule de Tzetzès (supra n. 11).

13 Déjà Euripide, Hippolyte, 161-169. 
commerce maritime (KOWALZIG, 2013, p. 194-196) ${ }^{14}$, en particulier en des zones dangereuses. Ainsi sur la longue côte rocheuse de l'île d'Ikaria, exposée aux vents du Nord-Ouest, le Tauropolion d'Oinoé, au fond d'un des rares abris, face à cette mer redoutée où s'était aussi abîmé, dans son aventureux voyage aérien, le fils de Dédale (Strabon, XIV, 1, 19 ; Anthologie Palatine, VII, 699) ${ }^{15}$. Plus au sud, à Patmos, une inscription d'époque impériale fait l'éloge de la jeune Véra, la noble fille du sage médecin Glaukias, native de l'île, qui a été choisie par ses compatriotes comme " hydrophore », c'està-dire prêtresse de l'Artémis Patmienne (SEG 39-855 ; MERKELBACH, STAUBER, 1998, 01/21/01, p. 169-170). La déesse y est aussi qualifiée de Scythienne ${ }^{16}$, car selon la tradition de " l'île très auguste de la fille de Létô », rappelée par l'inscription, c'est là que « le belliqueux Oreste l'avait ramenée du pays des Scythes pour l'y installer, mettant fin ainsi à son horrible folie de matricide (pausamenos stugerès mètrophonou maniès) ${ }^{17} \gg$. La déesse a son siège, hedrana, son temple, auquel s'est substitué le monastère de Saint Jean le Théologien, au sommet de l'île, et, de là, « la protège des abîmes de la mer ", tremblements de terre, tsunamis, ou sans doute, plus banalement, pirates (SAFFREY, 1975, p. 405-406, avec la traduction) ${ }^{18}$. C'est sur l'ordre de cette Artémis Scythienne (boulaisi Artémidos Skuthiès) que Véra a quitté la maison de son père, à Lébédos, sur la côte asiatique, au nord d'Éphèse, où exerçait le médecin et où elle avait été élevée. Pour rejoindre Patmos, courageusement, elle « a traversé les flots périlleux de la mer Égée pour célébrer avec l'éclat que prescrit la loi divine, la fête et le repas sacré ». Sur l'île voisine de Léros, la Parthénos veillait sur la rade probablement la mieux protégée de ce secteur des îles milésiennes (PATON, 1894).

Or, si au retour apaisé d'Oreste vers Athènes correspond l'institution d'un rituel apaisé, on ne peut toujours en dire autant des connotations des

\footnotetext{
14 On ne peut toutefois pas se fonder sur les mentions formulaires de la Tauropole dans les serments imposés aux cités par les dynastes ou rois macédoniens, qui marquent simplement les manifestations de la domination macédonienne (BRULÉ, 2007, XV, 38-41).

15 KOWALZIG, 2013, p. 194, avec vue générale du site, fig. 4. Pour les fouilles archéologiques, avec bibliographie antérieure, cf. ANGLIKER, à paraître. Dans l'ile de Failaka du golfe Persique, nommée Ikaros par Alexandre en l'honneur d'Icare, Artémis était qualifiée de Tauropole selon Strabon (XVI, 3, 2 ; cf. Arrien, Anabase, VII, 20, 3), et par l'épigraphie de Sôteira, avec, dans son sanctuaire sur le rivage, des offrandes de bateaux de terre cuite (FENET, 2016, p. 212-213 et 383-384).

$16 \mathrm{Au}$ total, la même Artémis reçoit dans cette inscription quatre épithètes : Parthenikè (Vierge), Elaphèbolos (Chasseresse), Patniè (de Patmos) et Skuthiè.

17 Pour le sens de l'expression et du verbe pauô dans le contexte, cf. ELLINGER, 2005, p. 143-170.

18 Passage (1. 8-9) de lecture et interprétation difficile ; voir aussi GRÜLL, 1987.
} 
autres versions du retour d'Oreste avec sa sœur et la petite statue. On a cherché à rendre compte de la tonalité de la version athénienne par le contexte d'un empire athénien encore confiant dans son destin et sa capacité à faire régner ordre et prospérité économique et commerciale en Égée (KOWALZIG, 2013, p. 199-210). Au contraire, les versions siciliennes de l'Artémis Phakélitis ou Lyaia, renvoient à un contexte de graves désordres, sinon de guerre civile, entre fin de l'archaïsme et guerres puniques (GRAF, 1979, p. 37-41 ; FRONTISI-DUCROUX, 1981). Dans la version rhodienne, la statue est installée dans le rempart de la ville insulaire qu'elle protègera des sièges, ce qui se comprend le mieux, sinon par rapport au fameux siège de Démétrios Poliorcète, en tout cas dans le contexte des multiples épiphanies d'Artémis au secours des cités de la région attaquées par les souverains hellénistiques (PETRIDOU, 2015, p. 130-138). Mithridate se prévaudra de l'appui de la Parthénos quand il se posera en défenseur des Grecs, à commencer par ceux de Chersonésos de Tauride et du Bosphore Cimmérien contre les Scythes, et de son iconographie particulièrement violente (la déesse frappe de sa lance un cerf dont elle brise le dos de son genou) dans sa propagande anti-romaine. Ce à quoi il faut relier ces autres prolongements des aventures d'Oreste, où, au lieu de revenir en Grèce, il se dirige au-delà du Pont Euxin vers l'Asie mineure orientale et, s'éloignant cette fois radicalement de la mer, installe la statue de Tauride sous les chaînes homonymes du Taurus à Comana du Pont, à Comana de Cappadoce, en Akilisène sur le Haut-Euphrate chez les Arméniens, ou encore à Hiérapolis Castabala de Cilicie ; elle y est identifiée aux déesses locales (Ma, Anahita), avec leurs rituels souvent réputés pour leur violence. Les généraux romains ne seront pas en reste, disputant à leurs adversaires les mêmes divinités, ou se revendiquant de ce qui pouvait le mieux chez eux leur être opposé, la Diane d'Aricie au bord du lac Némi, apportée elle aussi de Scythie par Oreste et qu'ils ont comblée de leurs bienfaits, avec leurs butins, avant que son image ne devienne à ce point, avec son sinistre rituel, indissolublement liée à leurs propres guerres civiles, qu'Auguste devra séparer Oreste de sa protectrice et l'enterrer sur le forum pour signifier le retour à la paix ${ }^{19}$.

19 Cf. GULDAGER BILDE, 2003, fondamental pour l'interprétation historique ; ELLINGER, 2009, ch. 6 : «Les retours de l'Artémis Taurique »; également BRAUND, 2018, p. 79-88. 
C'est dans ce contexte historique qu'il faut comprendre l'autre plus célèbre version de ce dossier, la version spartiate, au lieu de l'enfermer dans un face à face atemporel avec la version d'Euripide. Celle qui constitue l'aition de la refondation de l'agôgé au $\mathrm{I}^{\mathrm{er}}$ siècle avant notre ère, et de la reprise en main, après des décennies de désordre, de la cité et de sa jeunesse, à un moment où les élites spartiates vont bientôt choisir d'entrer dans l'ordre impérial (ELLINGER, 2011, p. 580-583). Ajoutons pour compléter ce trop rapide survol que le rituel qu'avait été chargée d'accomplir l'innocente Véra quand elle avait été rappelée dans sa patrie, avait quelque chose de passablement « scythique » : sacrifier à côté de l'autel de la déesse les fœtus des chèvres pleines que l'on venait d'égorger (BREMMER, 2005, p. 161). Et ce n'étaient sans doute pas seulement les flots qu'elle avait eu à traverser qui étaient agités, si l'on a raison de dater l'inscription du temps de la réaction païenne de l'empereur Julien (HALL, 2013, p. 143). Dix ans après la mort de ce dernier, à Andrinople, en 378, la déesse scythique d'Oreste, apportée là aussi par le héros, toujours assoiffée de sang, aurait sa dernière revanche, si l'on en croit l'ironie grinçante de l'auteur (païen) de la Vie d'Élagabal, dans l'Histoire Auguste (Histoire Auguste, Élagabal, VII, 5-9 ; ELLINGER, 1999, p. 35-36), avec le désastre de l'armée impériale face aux Goths et la mort de l'empereur (chrétien) dans les flammes de son refuge.

\section{Héra reste à Samos}

Au récit d'Oreste portant la statue d'Artémis Taurique en Laconie a souvent été associé un autre récit concernant la statue de l'Héra de Samos. La statue apportée par Oreste avait été en effet ensuite trouvée dans un buisson de gattilier (lugos) dont les branches l'encerclant la faisaient tenir toute droite, d'où le double nom qui lui sera donné, Orthia, qui se tient droite, et Lugodesma, liée par le gattilier. Comme le rapporte Pausanias, c'est cette statue qui avait d'abord provoqué la folie chez ceux qui l'avaient trouvée, puis, quand on lui avait élevé un autel et voulu lui sacrifier, des querelles sanglantes entre les habitants des subdivisions territoriales de la cité, et, en plus, elle avait envoyé la peste. Pour apaiser la guerre civile et le loimos, on avait dans un premier temps, pour obéir à l'oracle consulté, qui 
prescrivait « d'ensanglanter l'autel de sang humain », instauré un rituel de sacrifice humain par tirage au sort. Finalement, Lycurgue lui aurait substitué la fustigation des éphèbes sur l'autel, celle qui était pratiquée vers la fin de l'époque hellénistique et sous l'empire, et qui pouvait aller jusqu'à la mort (Pausanias, III, 16, 7-11).

Le motif de la guerre civile et de la peste rapproche l'histoire des versions siciliennes ; simplement ici la déesse n'en débarrasse pas la cité, mais il sert d'étape intermédiaire vers l'établissement de l'ordre (néo) lycurguéen de la Sparte romaine. Pour ce qui est de la ressemblance avec le récit samien ${ }^{20}$, le motif commun est celui du lugos qui tient la statue, mais le reste de la narration est fort différent. L'affaire se passe du temps d'Héraclès, puisque l'héroïne au centre de l'histoire est Admète, la fille d'Eurysthée qui avait ordonné les fameux Travaux. Elle est fâchée avec son père pour des raisons qui se sont perdues ${ }^{21}$ et s'enfuit d'Argos, et traverse l'Égée pour se réfugier à Samos, chez les Cariens. Là, la déesse Héra lui apparaît, et voulant la remercier pour avoir assuré son salut dans sa fuite, Admète décide de prendre en charge le culte dans le sanctuaire qu'avait fondé la population primitive de l'île, les Lélèges. Pour lui porter tort aux yeux des habitants, les Argiens engagent des pirates tyrrhéniens pour aller enlever la statue de la déesse. Ceux-ci débarquent de nuit, gagnent le sanctuaire proche du rivage, et sans difficultés, car il était si primitif qu'il n'avait même pas de portes, s'emparent de la statue et la portent sur le bateau. Mais quand ils veulent appareiller, ils ont beau s'acharner sur les rames, le navire reste obstinément immobile. Comprenant qu'il s'agit de l'action de la déesse, terrifiés, ils reportent la statue sur le rivage où ils la laissent entourée d'offrandes de galettes de la nourriture du bord, pour se faire pardonner, et ils se réembarquent et s'enfuient sans demander leur reste. Les Cariens du lieu, avec Admète, se sont aperçus de la disparition de la statue ; ils la cherchent partout et finalement la trouvent où les pirates l'ont laissée. Ils croient qu'elle s'est sauvée d'elle-même et pour éviter qu'elle ne recommence à fuguer,

20 Ménodote de Samos (fin IIIe s. avant J.-C.), Choses remarquables de Samos, 541 F 1 Jacoby = Athénée, XV, 671e-674a ; cf. DILLERY, 2005, p. 510-513.

21 Refus du mariage ? Son nom veut dire «Indomptée », et c'est elle qui désirait la ceinture donnée par Arès à la reine des Amazones, Hippolyte. D'où l'ordre donné par son père à Héraclès pour aller la chercher (Apollodore, Bibliothèque, II, 5, 9). Par ailleurs, elle est attestée comme prêtresse d'Héra à Argos pendant 58 ans, d'après la Tabula Albana $\left(\mathrm{I}^{\mathrm{er}} \mathrm{s}\right.$. avant J.-C.), plaque de marbre inscrite et illustrée montrant les exploits d'Héraclès ( $B N J 40$ F 1D ; IG XIV, 1293). Il nous manque manifestement des éléments. 
la ligotent avec des rameaux de gattilier. C'est là le motif commun avec l'histoire spartiate. La fille d'Eurysthée survient à son tour sur la plage, comprend l'erreur des barbares Cariens, libère la statue de ses entraves, et la réinstalle dans son temple. La déesse ne tiendra pas vraiment rigueur à ses fidèles trop zélés : un oracle les condamnera seulement à porter une couronne de rameaux de gattilier dans les sacrifices.

On a beaucoup spéculé sur les valeurs ambiguës du gattilier (agnus castus), anaphrodisiaque d'un côté, favorisant la fécondité de l'autre, qui pourraient convenir selon leur versant, aussi bien à la chaste Artémis qu'à la déesse du mariage. En revanche, le reste de l'histoire a été souvent négligé par les interprètes alors que tout son côté maritime ${ }^{22}$ concernant l'enlèvement de la statue mérite d'être comparé à l'histoire d'Oreste et Iphigénie et de la statue de Tauride. Ici, l'héroïne est prêtresse volontaire de la déesse et non, contre son gré, chargée d'exécuter des sacrifices qui lui répugnent. Oreste agissant sur l'ordre d'Apollon s'oppose évidemment aux pirates tyrrhéniens, qui seraient plus proches des Taures comme pirates et ennemis des Grecs. La facilité à s'emparer de la statue d'Héra logée dans un sanctuaire si primitif et tout proche d'un rivage plat ${ }^{23}$, sans aucune protection, contraste avec le temple, au sommet de sa falaise, des redoutables Taures, entouré de hauts murs, avec de solides portes de bronze. Finalement, alors qu'Artémis ne manifeste aucune opposition à son enlèvement, mais que l'obstacle qui manque de tout faire échouer vient des éléments - le vent et les vagues contraires - sans qu'on puisse les attribuer à l'action d'un dieu, ici sur une mer parfaitement calme où rien ne s'oppose au départ, c'est la seule action de la déesse qui immobilise le bateau, le lie en quelque sorte, alors que les amarres ont été déliées et les ancres levées. ${ }^{24} \mathrm{Il}$ faudra donc laisser sur place la statue, là où Oreste pourra l'emporter avec l'aide d'Athéna et Poséidon.

\footnotetext{
22 Pour le gattilier, c'est plutôt son côté lieur qui est explicitement mis en valeur ici, comme le note à juste titre VON STADEN, 1993, p. 48-51. Voir aussi les remarques de PIRENNE-DELFORGE, PIRONTI, 2016, p. 142-152, 154-155, qui n'abordent qu'avec beaucoup de prudence l'aspect maritime du récit. Au contraire, ROMERO RECIO, 2010, p. 95-100 ; FENET, 2016, p. 72-74.

23 C'est sur le chemin, entre le rivage et le temple, que les Samiens de l'époque archaïque empruntaient pour venir honorer la déesse, s'y rendant à l'époque en bateau depuis la ville, qu'ont été retrouvées les offrandes de modèles en bois de navires (KYRIELEIS, 1993, p. 107, 112).

24 Les uns et les autres s'acharnent en vain sur les rames (cf. Euripide, I.T., 1386-1397), mais les pirates ne rament contre aucun vent ni aucune vague.
} 
Héra ne veut pas fuir, comme l'avait bien compris Admète dont elle avait protégé la fuite sur terre et sur mer. ${ }^{25}$ Artémis, au contraire, a fui avec Oreste et Iphigénie, mais la déesse peut aussi se laisser enlever dans des contextes qui ne semblent pas pour elle une libération. Ne parlons pas de la statue de Brauron - là encore l'Artémis Taurique d'Oreste, selon les Athéniens du temps de Pausanias - qui a été enlevée par les Perses pendant leur invasion de l'Attique lors de la deuxième guerre médique, et emportée à Suse. Séleucos l'y avait redécouverte, pour l'offrir aux citoyens de sa nouvelle fondation de Laodicée de Syrie, où elle avait retrouvé la mer et des rituels sanglants - si l'on en croit Porphyre : le sacrifice annuel d'une jeune fille à laquelle aurait été ultérieurement, sous Hadrien, substituée une biche, en une reprise de la séquence d'Aulis (Porphyre, De l'abstinence, II, 56, 3-4). En tout cas, pour Pausanias, cet enlèvement est bien la preuve que cette statue de Brauron, pas plus que celles de Lydie, de Cappadoce ou du Pont, n'est la véritable statue apportée par Oreste et Iphigénie (qui pour lui est celle d'Orthia). Si cela avait été le cas, les Athéniens n'auraient pas manqué de l'emporter avec eux sur leurs bateaux (!) quand ils ont évacué l'Attique (Pausanias, I, 33, 1 et III, 16, 7-8).

\section{Errances de Brauron à Lyktos}

Pourtant, il y a encore à Brauron une autre statue d'Artémis qui aura été enlevée et qui va avoir à son tour un long parcours maritime plein de péripéties. L'affaire concerne la fondation de la grande cité crétoise de Lyktos et se déroule entre l'île de Lemnos au nord de l'Égée, Brauron, Sparte et finalement la Crète. Elle est racontée principalement par Plutarque dans son traité des Vertus des femmes $(8,247 \mathrm{a}-\mathrm{f})$ et dans ses Questions grecques (21, 296b-d) (MALKIN, 1994, p. 76-80 ; FENET, 2016, p. 205-206), mais a été, selon toute apparence, contaminée par d'autres récits à la fois du cycle lemnien et de la colonisation lacédémonienne en Méditerranée. Tout

25 Les termes employés (tès oikothen sôtèrias charisterion, « en remerciement du salut [dans sa fuite] depuis chez elle ») impliquent assurément les dangers de la traversée. 
commence à Brauron quand les Pélasges ou Tyrrhéniens de Lemnos et d'Imbros enlèvent, sur leurs pentécontères, les femmes et/ou les filles des Athéniens venues honorer la déesse ${ }^{26}$. Soit pour se venger des Athéniens qui les avaient à leurs yeux injustement expulsés d'Attique (Hérodote, VI, 137, donne les points de vue opposés des Lemniens et des Athéniens), soit parce qu'ils étaient dès l'origine des pirates violents et nuisibles (Philochore, 328 F 100 et 101, d'où leur ethnique également de Sintiens, " Malfaisants »). Pour mener cette « embuscade» (elochèsan), les Pélasges profitaient de leur connaissance, sans doute acquise lors de leur séjour attique, de la date de la fête (Hérodote, VI, 138) ${ }^{27}$ - et de la vulnérabilité du lieu, en bord de mer et très loin de la ville. Ils prirent les Athéniennes enlevées pour femmes et en eurent des enfants. Plus tard, les Athéniens s'emparèrent des îles en question et en expulsèrent la population, descendant de ces unions, sous le prétexte qu'il s'agissait de demi-barbares (mixobarbarous - Vertus des femmes, 247a). Les Tyrrhéniens se réfugièrent au Ténare, sans doute déjà bien connu des pirates, et offrirent leurs services aux Spartiates dans la guerre contre les Hilotes. Le récit de Plutarque reproduit alors en le résumant celui bien connu d'Hérodote concernant les Minyens de Lemnos (Hérodote, IV, 145148), à ceci près que pour Hérodote ces Minyens sont les descendants des Lemniennes (qui avaient tué tous leurs hommes qui leur préféraient leurs captives thraces) et des Argonautes. Et ils avaient été chassés de Lemnos par les Pélasges qui enlèveraient les femmes athéniennes à Brauron! De toute façon, ici, l'histoire des enfants des unions entre Pélasges et filles d'Athènes, prises comme concubines et non comme femmes légitimes, tourne court, puisque les Pélasges alarmés par l'intelligence et les capacités, venant de leurs mères athéniennes (!), de cette progéniture bâtarde, par rapport à celles de leurs enfants légitimes, s'empressent de tous les massacrer.

Passons rapidement sur l'histoire de ces Minyens/Tyrrhéniens, d'abord bien accueillis dans la cité spartiate avec droit d'intermariage, puis trop exigeants, réclamant accès au Conseil et aux magistratures, et même à la royauté, leur complot et arrestation. La nuit où ils vont être exécutés, leurs femmes spartiates obtiennent le droit d'une dernière visite et échangent avec

26 Femmes : Hérodote, VI, 138 ; Plutarque, Vertus des femmes, 247b et Questions grecques, $296 \mathrm{~b}$ (femmes et filles). Filles seulement : Philochore, BNJ 328 F 100, «parthenoi venues 'faire l'ourse' pour la déesse aux Brauronies » et F 101, « jeunes filles canéphores ». De toute façon, d'après les documents archéologiques, femmes adultes, adolescentes et petites filles participaient en divers rôles aux rituels d'époque classique. 27 Philochore, 328 F 100, précisera qu'il s'agit des Brauronies. 
eux leurs vêtements. Les Spartiates sont alors contraints de négocier avec les évadés qui tiennent la montagne et menacent maintenant de s'allier aux hilotes. Ils les autorisent à repartir, avec leurs femmes spartiates, pour fonder une colonie là où ils le pourront, leur désignant aussi des oikistes spartiates, Pollis, Delphos et Krataïdas, ce qui leur donnera ensuite le titre de colons de Lacédémone. Une partie de la troupe colonisera l'île de Mélos, mais le reste, conduits par Pollis et Delphos, pour obéir à l'oracle de Delphes de rigueur, non encore réalisé, poursuivent leurs navigations incertaines. Elles les mènent vers le centre de la côte nord de Crète, où ils débarquent et campent près de Chersonésos. Or, la nuit, survient une peur panique. Épouvantés, ils sautent dans leurs navires, oubliant à terre le xoanon, la petite statue en bois d'Artémis qui les accompagnait dans toutes leurs pérégrinations, celle que leur avaient transmise leurs ancêtres et que ceux-ci avaient enlevée en même temps que les femmes à Brauron. Une fois en mer, comme le trouble avait cessé, et qu'ils voulaient reprendre leur navigation, ils s'en aperçurent et s'en lamentaient, et, en même temps, Pollis découvrit qu'ils avaient aussi perdu la pointe de leur ancre qui s'était apparemment arrachée sur les rochers du fond, sans qu'ils s'en rendent compte dans leur précipitation. Il comprit alors et déclara que les prédictions étaient réalisées. Le dieu avait en effet prophétisé que « leur errance cesserait (pausasthai planès) lorsqu'ils perdraient leur déesse et leur ancre, et de fonder là leur cité ». Il ordonna donc de retourner à terre, et ils s'emparèrent du pays à travers de multiples combats, fondant Lyktos et soumettant à leur domination de nombreuses autres cités (Vertus des femmes, 247d-f).

On voit ici que le récit dramatise dans une nouvelle combinaison le moment décisif de l'embarquement, tout comme l'Iphigénie en Tauride et le récit samien : le moment où les ancres levées et les amarres déliées, le navire d'Oreste dérive dangereusement, en même temps qu'Iphigénie a peur d'entrer dans l'eau et que son frère doit la prendre, avec la statue, sur son épaule, tandis que les Taures cherchent à s'emparer des câbles pas encore entièrement remontés et que les archers du bord les repoussent à coups de flèches; le moment aussi où alors que tout semble se passer parfaitement pour eux, amarres et ancres remontées, la statue à bord, les pirates tyrrhéniens à Samos ne parviennent pas faire s'éloigner le navire du rivage. Ici, la statue restée sur le rivage, désigne dans cet oubli parlant, cette terre comme celle promise par le dieu, et où ils devront s'ancrer en la conquérant (FENET, 2016, 
p. 205).$^{28} \mathrm{Il}$ est aussi remarquable qu'elle a assuré une protection continue, restant solidaire avec elles, sans jamais les abandonner, aux femmes et aux jeunes filles, ses fidèles, avec lesquelles elle avait été enlevée, ainsi qu'à leur descendance, même " mixobarbare ", dans toutes ses pérégrinations et vicissitudes. On peut penser aussi qu'elle a étendu cette protection aux héroïques Lacédémoniennes qui avaient choisi de partager tous les risques avec eux ${ }^{29}$, avant de donner l'indication décisive à l'expédition coloniale du lieu où s'établir. Au total, divinité à la fois ancestrale et intégratrice, elle confère au nouveau peuple des Lyktiens cette identité multiple dont il s'enorgueillissait, « se considérant parents des Athéniens par la race (kata genos), grâce à leurs mères, et colons des Spartiates ».

Il est probable aussi, cette histoire, marquée dès l'origine par une aura de violence, n'étant pas finie, que la déesse a continué à soutenir les nouveaux Lyktiens dans leur conquête du territoire, dans des guerres dont ce qui subsiste de cette tradition nous indique qu'elles eurent aussi un côté que l'on pourrait dire artémisiaque, transgressant les limites admises ordinairement entre Grecs. Dans les combats, leurs adversaires crétois ne permettaient pas aux Lyktiens de relever leurs morts. Pollis dut alors instituer un groupe de citoyens doté de privilèges spéciaux, les "Brûleurs ", qui avaient pour tâche d'aller plus tard recouvrer les corps en décomposition et de leur donner en les brûlant les honneurs rituels. ${ }^{30}$ Enfin, la fin du récit rejoignant le début, l'autre oikiste, Delphos, ayant, en bonne entente, fondé une autre cité « tyrrhénienne », les anciens pirates passèrent entre Tyrrhéniens de Crète des accords (philanthrôpa) excluant les actes injustes de pillage et de brigandage qui sont la coutume des Crétois entre eux (296d). Le point est intéressant. Le récit flottait singulièrement dans sa chronologie entre l'Athènes la plus ancienne, la conquête dorienne, les diverses guerres de Messénie, la conquête de Lemnos et d'Imbros par Miltiade. Il se termine en aition des asylies accordées par les cités crétoises au temps de leurs guerres et pirateries de l'époque hellénistique, nous donnant le contexte d'énonciation le plus probable de la source de Plutarque concernant la fondation de Lyktos.

28 Le redoublement du message est essentiel et désigne sans doute aussi Chersonésos, appelée parfois Lyktos sur mer, comme port de Lyktos. Son culte principal était celui de l'Artémis du centre de la Crète, Britomartis. $29 \mathrm{Ou}$, si l'on préfère, que la tonalité artémisiaque de l'histoire (risque, héroïsme féminin, travestissement sexuel), justifie le collage des récits.

30 C'est l'objet même de la Question grecque 21, 296b-c («Qui appelle-t-on « Brûleurs » chez les Crétois ?»). 
Une dernière histoire, rapportée par Pausanias dans son livre sur la Laconie pourrait être rattachée à ce contexte hellénistique, malgré son habillage mythologique. Le Lacédémonien Knageus avait participé à l'expédition des Dioscures contre Aphidna en Attique, pour récupérer leur sœur Hélène qui avait été enlevée par Thésée, alors que petite fille elle dansait au sanctuaire d'Artémis Orthia. Il fut fait prisonnier par les Athéniens et vendu en Crète, où il devint l'esclave d'un sanctuaire d'Artémis. Il réussit à convaincre la jeune fille qui était la prêtresse de s'enfuir avec lui, par mer évidemment, en emportant la statue de la déesse. Arrivés à Sparte, ils fondèrent le sanctuaire d'Artémis Knagia (Pausanias, III, 18, 4). ${ }^{31}$

On a cherché ici à prendre une vision large d'un groupe de récits qui se déploie sur un très vaste arc chronologique. Il faut cesser de considérer qu'il n'y a de « mythe » vivant et digne d'intérêt qu' « archaïque ». Au contraire, il est nécessaire de considérer ces récits dans leurs contextes d'énonciation et de réception, de reformulation ou de création, des différentes époques, qu'elles soient pré-classique, classique, hellénistique, ou romaine jusqu'à l'Antiquité tardive. Revenons pour terminer au récit samien de l'enlèvement de la statue d'Héra. A quel moment historique un tel récit pouvait-il le plus faire sens sous son aspect maritime ? Non pas aux temps de la piraterie crétoise hellénistique où les cités menacées et leurs sanctuaires cherchaient à obtenir des traités d'asylie des Crétois ou des Étoliens. Héra ici n'a pas besoin de cela. Plutôt à la grande époque de la cité et du sanctuaire, où les aristocrates samiens consacraient la dîme de leur butin sur mer (sulèn) à la déesse, comme Aiakès (IG XII, 6, 2, 561) 32 $^{32}$, peut-être le père de Polycrate, et ne rougissaient pas de porter des noms comme Syloson («Qui garantit les prises en mer »), son frère, et où le tyran de Samos recommandait à ses équipages de s'emparer aussi bien des navires amis qu'ennemis, quitte à

31 On rejoint ici les histoires étudiées dans ELLINGER, 2011, dont j'ai montré le contexte de signification hellénistique et romain. L'Artémis du Limnaion de Sparte (Orthia), dans la Sparte conquise par les Doriens, persuade le dernier représentant de la légitimité achéenne de voler sa statue, avec son fils enfant, et d'aller avec elle fonder Patras. Mais le voyage se fait par terre, la statue étant portée par le fidèle esclave des fondateurs. Les vicissitudes des statues continuaient à générer des récits : lors du sac de Délos en 88 avant J.-C., un soldat barbare de Ménophanès, l'amiral de Mithridate, jette à la mer un xoanon d'Apollon. Les flots le portent sur la côte égéenne de la Laconie. Les habitants lui consacrent un sanctuaire sur le rivage, là où ils l'avaient recueilli, et nomment le lieu Épidélion, « Nouvelle Délos » (Pausanias, III, 23, 2-5 ; cf. F. Prost dans ÉTIENNE, 2017, p. 182-183). Apollon se vengea : des marchands italiens, survivants du massacre à Délos, coulèrent le navire de Ménophanès. Et plus tard le dieu provoqua la mort de Mithridate.

32 Cf. Léon de Samos, historien du $\mathrm{II}^{\mathrm{e}}$ s. avant J.-C. écrivant les exploits des Samiens sur leurs navires et comment ils ont orné le sanctuaire d'Héra de leurs butins (BNJ 540 T 1 ; DILLERY, 2005, p. 512-513). 
rendre ensuite leurs biens aux amis qui n'en seraient que plus reconnaissants (Hérodote, III, 39, 3-4). L'époque encore où les adversaires samiens de Polycrate s'emparent de Kydonia en Crète et construisent le sanctuaire du Diktynnaion, tout en écumant la route du commerce vers l'Occident dans le détroit entre Crète et Péloponnèse. Les Éginètes, grands rivaux des Samiens de tous bords politiques, les mirent hors d'état de nuire et consacrèrent les proues de leurs navires qu'ils avaient capturés au sanctuaire d'Aphaia, forme locale d'Artémis, de Britomartis ou de Diktynna (Hérodote, III, 59). Pindare peut faire l'éloge d'Égine qui « règne sur la mer dorienne » (Pindare, Péans, VI, 123 [medeoisan] et 130 [nauprutanin]). À Samos, ici aussi, nous avons une Héra Reine, de l'île ${ }^{33}$ et de la mer.

\section{Bibliographie}

ANGLIKER, Erica. The Cycladic Archipelago beyond geographical definitions: redefining boundaries and limits through material culture and religion (à paraître).

BIELFELDT, Ruth. Orestes auf römischen Sarkophagen. Berlin: Reimer, 2005.

BRAUND, David. Parthenos and the Nymphs at Crimean Chersonesus: colonial appropriation and native integration. In: BRESSON, Alain, IVANTCHIK, Askold, FERRARY, Jean-Louis (eds.). Une koinè pontique : cités grecques, sociétés indigènes et empires mondiaux sur le littoral nord de la mer Noire (VII s. a.C. $-I I I^{\mathrm{e}}$ s. p.C.). Bordeaux: Ausonius, 2007, p. 191-200.

. Greek Religion and Cults in the Black Sea Region: Goddesses in the Bosporan Kingdom from the Archaic Period to the Byzantine Era. Cambridge: Cambridge University Press, 2018.

BREMMER, Jan N. The sacrifice of pregnant animals. In: ALROTH, Brita, HÄGG, Robin, (eds.). Greek Sacrificial Ritual, Olympian and Chthonian. Stockholm: Astrom, 2005, p. 155-165.

33 Nèsou despotin, pour le poète samien Nicainétos, cité par Athénée dans le même passage que précédemment $(\mathrm{XV}, 673 \mathrm{~b})$. 
BRULÉ, Pierre. La Grèce d'à côté : réel et imaginaire en miroir en Grèce antique. Rennes: Presses universitaires de Rennes, 2007. https://books. openedition.org/pur/6212.

CALAME, Claude. Le rite d'initiation tribale comme catégorie anthropologique (Van Gennep et Platon). Revue de l'histoire des religions, 220, 2003, p. 5-62.

DILLERY, John. Greek sacred history. American Journal of Philology, 126, 2005, p. 505-526.

ELLINGER, Pierre. Guerres d'anéantissement, récits de guerre d'anéantissement. Pallas, revue d'études antiques, 51, 1999, p. 25-38.

. La fin des maux. D’un Pausanias à l'autre. Paris: Les Belles Lettres, 2005.

. Artémis, déesse de tous les dangers. Paris: Larousse, 2009.

. Le maître et son fidèle esclave : Artémis Limnatis et l'identité de la cité de Patras. In: BREGLIA, Luisa, MOLETI, Alda, NAPOLITANO, Maria Luisa (eds.). Ethne, identità e tradizioni: La terza Grecia e l'Occidente. Pisa: ETS, 2011, p. 573-586.

. Fondation de la cité et réconciliation des sexes à Milet : entre Artémis Chitônê et Aphrodite. In: AZOULAY, Vincent, GHERCHANOC, Florence, LALANNE, Sophie (eds.). Le banquet de Pauline Schmitt Pantel : genre, mours et politique dans l'Antiquité grecque et romaine. Paris: Publications de la Sorbonne, 2012, p. 225-240.

ÉTIENNE, Roland. Le sanctuaire d'Apollon à Délos. I. Architecture, topographie, histoire. Athènes: École française d'Athènes, 2017.

FENET, Annick. Les dieux olympiens et la mer : espaces et pratiques cultuelles. Rome: École française de Rome, 2016.

FRONTISI-DUCROUX, Françoise. Artémis bucolique. Revue de l'histoire des religions, 198, 1981, p. 29-56.

GRAF, Fritz. Das Götterbild aus dem Taurenland. Antike Welt, 10, 1979, p. 33-41.

GRÜLL, Tibor. Patmiaka. Two studies on ancient Patmos. Specimina nova dissertationum ex Instituto Historico Universitatis Quinqueecclesiensis de Iano Pannonio nominatae, 1, 1987, p. 15-67. 
GUARISCO, Diana. Santuari "gemelli" di una divinità: Artemide in Attica. Bologna: Bononia University Press, 2015.

GULDAGER BILDE, Pia. Wandering images: from Taurian (and Chersonesean) Parthenos to (Artemis) Tauropolos and (Artemis) Persike. In: GULDAGER BILDE, Pia, HØJTE, Jakob Munk, STOLBA, Vladimir F. (eds.). The Cauldron of Ariantas: Studies presented to A. N. Ščeglov on the occasion of his 70th birthday. Aarhus: Aarhus University Press, 2003, p. 165-183.

HALL, Edith. Adventures with Iphigenia in Tauris: A Cultural History of Euripides' Black Sea Tragedy. Oxford: Oxford University Press, 2013.

KOWALZIG, Barbara. Transcultural chorality: Iphigenia in Tauris and Athenian imperial economics in a polytheistic world. In: GAGNÉ, Renaud, GOVERS HOPMAN, Marianne (eds.). Choral Mediations in Greek Tragedy. Cambridge: Cambridge University Press, 2013, p. 178-210.

KYRIAKOU, Poulheria. A Commentary on Euripides' Iphigenia in Tauris. Berlin: de Gruyter, 2006.

LEITAO, David D., "Solon on the beach: some pragmatic functions of the limen in initiatory myth and ritual". In: PADILLA, Mark W. (ed.). Rites of Passage in Ancient Greece: Literature, Religion and Society. Lewisburg PA: Bucknell University Press, 1999, p. 247-277.

MALKIN, Irad. Myth and Territory in the Spartan Mediterranean. Cambridge: Cambridge University Press, 1994.

. A Small Greek World: Networks in the Ancient Mediterranean. Greeks Overseas. Oxford: Oxford University Press, 2011.

MERKELBACH, Reinhold, STAUBER, Josef. Steinepigramme aus dem griechischen Osten, I. Stuttgart, Leipzig: B.G. Teubner, 1998.

PATON, W.R. Calymna and Leros. Classical Review, 8,1894, p. 375-377.

PETRIDOU, Georgia. Divine Epiphany in Greek Literature and Culture. Oxford: Oxford University Press, 2015.

PIRENNE-DELFORGE, Vinciane, PIRONTI, Gabriella. L'Héra de Zeus : ennemie intime, épouse définitive. Paris: Les Belles Lettres, 2016. 
ROMERO RECIO, Mirella. Extrañas ausencias: las fiestas marítimas en el calendario litúrgico griego. Dialogues d'histoire ancienne, 36, 2010, p. 51-117.

SAFFREY, Henri-Dominique. Relire l'Apocalypse à Patmos. Revue biblique, 1975, p. 385-417.

SALIOU, Catherine. Statues d'Antioche de Syrie dans la Chronographie de Malalas. In: AGUSTA-BOULAROT, Sandrine, BEAUCAMP, Joëlle, BERNARDI, Anne-Marie, CAIRE, Emmanuelle (eds.). Recherches sur la Chronique de Jean Malalas, II. Paris: Centre de recherche d'histoire et civilisation de Byzance, 2006, p. 69-95.

USTINOVA, Yulia. The Supreme Gods of the Bosporan Kingdom. Leiden: Brill, 1999.

VON STADEN, Heinrich. Spiderwoman and the chaste tree. Configurations, 1, 1992, p. 23-56.

RECEBIDO EM: 19/06/2020 APROVADO EM: 25/08/2020 